\title{
Medullomyoblastoma with Myogenic Differentiation
}

National Cancer Institute

\section{Source}

National Cancer Institute. Medullomyoblastoma with Myogenic Differentiation. NCI

Thesaurus. Code C3706.

A rare malignant embryonal neoplasm arising from the cerebellum. It is characterized by the morphologic features of a medulloblastoma and the presence of a striated muscle component. Its clinical behavior is similar to medulloblastoma. 